\section{Multimodal imaging including spectral- domain optical coherence tomography and confocal near- infrared reflectance for characterization of lacquer cracks in highly myopic eyes}

C-F Liu', ${ }^{1,2}, \mathrm{LLiu}^{1,3}, \mathrm{C}-\mathrm{C} \mathrm{Lai}^{1,3}, \mathrm{JCL} \mathrm{Chou}^{4}$, L-K Yeh ${ }^{1,2}$, K-J Chen ${ }^{1,3}$, Y-P Chen ${ }^{1,3}$, W-C Wu ${ }^{1,3}$, L-H Chuang ${ }^{1,2}$, C-C Sun ${ }^{1,2}$ and N-K Wang ${ }^{1,3}$

\begin{abstract}
Purpose To compare multimodal imaging in detecting lacquer cracks in highly myopic eyes, and to correlate these findings with those of spectral-domain optical coherence tomography (SD-OCT).

Methods An observational case series study. Patients with a refractive error worse than

-8 diopters and lacquer cracks were recruited. The rates of detection of the lacquer cracks using multimodal imaging including near-infrared reflectance (NIR) imaging, fundus autofluorescence (FAF) imaging, and fluorescence angiography (FA) were compared. The characteristic findings of multimodal imaging were correlated with those of SD-OCT.

Results NIR imaging was more sensitive (92.9\%) in detecting lacquer cracks than either FAF $(12.5 \%)$ or FA $(67.9 \%)$. Lacquer cracks showed hyperreflectance on NIR, and they were consistently associated with a continuous retinal pigment epitheliumBruch's membrane complex, thinner choroid, and acoustic shadows on SD-OCT. Conclusions NIR imaging is superior to blue laser light (FAF and FA) imaging in detecting lacquer cracks. SD-OCT in combination with NIR located primary pathological lacquer cracks in the intact retinal pigment epithelium-Bruch's membrane complex as well as thinner choroid. These findings indicate that
\end{abstract}

multimodal cSLO and SD-OCT imaging allow for detecting of lacquer cracks in highly myopic eyes.

Eye (2014) 28, 1437-1445; doi:10.1038/eye.2014.221; published online 19 September 2014

\section{Introduction}

Myopic maculopathy is one of the most common causes of irreversible blindness in East Asia because the prevalence of severe myopia is high. ${ }^{1,2}$ Research performed by Hayashi et al ${ }^{3}$ on the long-term progression of myopic maculopathy suggested that $13.3 \%$ of eyes with lacquer cracks develop choroidal neovascularization (CNV), and $42.7 \%$ progress to patchy chorioretinal atrophy. Ohno-Matsui and Tokoro also reported that a large proportion of lacquer cracks progressed to advanced fundus changes during a mean follow-up period of 6 years. ${ }^{4}$ Therefore, the early detection of lacquer cracks in highly myopic eyes is crucial, because their formation is considered to be the step immediately preceding the development of CNV (wet-type myopic maculopathy) and patchy chorioretinal atrophy (severe dry-type myopic maculopathy)., $35-8$ Both myopic CNV and patchy chorioretinal atrophy are severe myopic maculopathies that result in irreversible visual loss.

Salzmann ${ }^{9}$ first described 'defects in the lamina vitrea' in high myopia in 1902.
${ }^{1}$ Chang Gung University College of Medicine, Taoyuan, Taiwan

${ }^{2}$ Department of Ophthalmology, Chang Gung Memorial Hospital, Keelung, Taiwan

${ }^{3}$ Department of Ophthalmology, Chang Gung Memorial Hospital, Linkou Medical Center,

Taoyuan, Taiwan

${ }^{4}$ Department of Ophthalmology, University of Ottawa, Ottawa, Ontario, Canada

Correspondence: N-K Wang, Department of Ophthalmology, Chang Gung Memorial Hospital, No. 5, Fu-Hsing Street, Kuei Shan, Taoyuan 333, Taiwan.

Tel: +886 33281200 , ext. 8666;

Fax: +886 33287798 .

E-mail: wang.nankai@ gmail.com

Received: 20 April 2014 Accepted in revised form: 12 August 2014 Published online: 19 September 2014 
The lamina vitrea is also referred to as 'Bruch's membrane'. Later, the term 'lacquer cracks' was used to describe this lesion, which typically occurs as yellowish-to-white lines in the posterior segment of highly myopic eyes, resulting from progressive eyeball elongation. ${ }^{6,10}$ Its prevalence ranges from $4.3-9.2 \%$ in highly myopic eyes. ${ }^{4,10,11}$ Several studies have reported that lacquer cracks represent linear breaks in Bruch's membrane and are associated with retinal hemorrhage and subretinal neovascularization. ${ }^{11-13}$ The histopathology of patients affected by lacquer cracks that can enable correlation with clinical presentation has rarely been reported. Grossniklaus and Green analyzed 308 eyes with pathological myopia and found breaks in Bruch's membrane in two eyes $(0.6 \%)$ with both lacquer cracks and subretinal neovascularization. ${ }^{11}$ However, there have been till now no histopathological studies on lacquer cracks without retinal hemorrhage or subretinal neovascularization.

To date, the best way to visualize lacquer cracks is with indocyanine green angiography (ICGA), which is an invasive examination. ${ }^{5,14}$ However, dye allergy is a concern when ICGA is used to detect lacquer cracks in highly myopic eyes. Multimodal confocal scanning laser ophthalmoscopy (cSLO) has recently become increasingly popular, and fundus autofluorescence (FAF), near-infrared reflectance (NIR), and spectral-domain optical coherence tomography (SD-OCT) imaging are noninvasive tests that can be used to visualize the pathological features of the retinal pigment epithelium (RPE), retina, and choroid. ${ }^{15,16}$

Although choroidal thickness, measured with SD-OCT, can predict the development of lacquer cracks, ${ }^{17}$ other types of topographic fundus imaging such as FAF and NIR, have not yet been used, and the value of these imaging modalities in identifying lacquer cracks remains unknown. Furthermore, whether or not noninvasive multimodal cSLO imaging allows for the detection and characterization of lacquer cracks in highly myopic eyes before an invasive ICGA examination remains unresolved. The purpose of this study was to compare the appearance of lacquer cracks on multimodal cSLO imaging, including FAF, NIR, FA, ICGA, and SD-OCT, and to investigate whether SD-OCT can reveal in vivo the 'optical' pathology that is detected by specific multimodal cSLO imaging.

\section{Patients and methods}

We conducted this observational case series study of patients with highly myopic eyes (defined as having a spherical equivalent refractive error worse than -8 diopters (D)) at Chang Gung Memorial Hospital, Linkou and Taipei from 1 June 2010 to 30 September 2013. This study was approved by the Institutional Review Board of Chang Gung Memorial Hospital, Taiwan (99-1061B) in 2010. Informed consent was obtained from all subjects. The procedures used conformed to the tenets of the Declaration of Helsinki.

Only highly myopic eyes with lacquer cracks (defined as typical hypofluorescent lines in late-phase ICGA) were enrolled in this study. Eyes with CNV, macular hemorrhage, severe cataract (which result in poor quality of multimodal imaging), amblyopia, glaucoma, uveitis, diabetic retinopathy, retinal vascular abnormalities, drusen, macular scarring, refractive surgery, or intraocular surgery (such as cataract extraction or vitrectomy) were excluded. The eyes with lacquer cracks were divided into linear or stellate groups using latephase ICGA applied for 20-40 min after the injection of the dye, as described previously. 5,8

\section{Basic and advanced examinations}

All of the eyes underwent a full ophthalmic evaluation, including best-corrected visual acuity (BCVA), slit-lamp examination, indirect ophthalmoscopy, measurement of the spherical equivalent refractive error (KR-8100 Autorefractor; Topcon, Tokyo, Japan), and axial length (A-Scan model A2500; Sonomed Inc., New York, NY, USA), as described previously. 8,17

Multimodal cSLO imaging including NIR, FAF, FA (10\% fluorescein sodium; Alcon Laboratories. Inc., Fort Worth, TX, USA) and ICGA (Diaichi Sankyo Propharma Co., Ltd, Tokyo, Japan) was performed with a confocal scanning laser ophthalmoscope (Heidelberg Retina Angiograph HRA2; Heidelberg Engineering, Dossenheim, Germany), as described previously, ${ }^{18}$ and SD-OCT images were obtained using a Spectralis HRAOCT system (Heidelberg Engineering). This allowed us to simultaneously obtain either NIR or FAF images with registered SD-OCT images. Enhanced depth imaging was used to improve the visualization of the choroid as described by Spaide et al. ${ }^{19}$

The HRA2 contains a small pinhole aperture, which suppresses light originating from outside the focal plane to enhance the image contrast compared with that of non-confocal imaging. It uses an argon blue laser light with a wavelength of $488 \mathrm{~nm}$ for excitation and a barrier filter of $500-700 \mathrm{~nm}$ to record FAF and FA. ${ }^{20}$ For NIR imaging, the excitation wavelength was $787 \mathrm{~nm}$, and the barrier filter allowed the passage of light at wavelengths $>810 \mathrm{~nm}$. Full-emission spectra were recorded with a polarization filter to obtain the NIR images.

To achieve better image resolution, each image had at least nine single 30-degree $\times$ 30-degree or 55-degree $\times$ 55 -degree images using an automatic real-time (ART) module (Heidelberg Engineering). The protocol for the 
SD-OCT scans included 100 high resolution frames for 9-mm line scans. The number of frames per B-scan was adjusted according to patient cooperation and fixation.

\section{Statistical analysis}

BCVA was measured on a Snellen chart and converted to the logarithm of the minimum angle of resolution (logMAR) for analysis. All data were analyzed using SPSS software (version 17.0; IBM, Armonk, NY, USA). Continuous covariates were assessed with an independent-samples $t$-test or the Mann-Whitney $U$-test, depending on the sample size in each group. Categorical covariates were assessed individually with the $\chi^{2}$ test, and Fisher's exact test was performed for samples with expected values of $<5$. A two-tailed $P$-value $<0.05$ was considered to be statistically significant.

\section{Results}

\section{Demographic data}

Thirty-four patients had lacquer cracks, including 22 patients with lacquer cracks in both eyes, three patients with lacquer cracks in one eye and CNV in the other, two patients with lacquer cracks in one eye and macular hemorrhage without $\mathrm{CNV}$ in the other eye, six patients with lacquer cracks in only one eye, and one patient with severe nuclear-type cataract that obscures the retinal imaging in one eye. In total, 56 eyes with lacquer cracks in 34 patients were included and analyzed in this study. Of the 56 eyes, 30 had stellate lacquer cracks, and 26 had linear lacquer cracks. Table 1 summarizes the demographic data of these 56 eyes. The mean age of the participants was $44.4 \pm 11.1$ years (mean \pm standard deviation, range 21-67 years). The mean refractive error was $-13.83 \pm 3.02 \mathrm{D}$ (range -9.13 to $-19.50 \mathrm{D}$ ), the mean axial length was $29.48 \pm 1.40 \mathrm{~mm}$ (range $26.48-33.23 \mathrm{~mm}$ ), and the mean LogMAR was $0.57 \pm 0.55$ (Snellen equivalent $0.44 \pm 0.33$ ).

\section{Detection of lacquer cracks with multimodal retinal imaging}

Table 2 shows the detection rates of lacquer cracks using different multimodal imaging methods. There were no statistically significant differences between the detection rates for linear and stellate lacquer cracks using the different types of multimodal retinal imaging (NIR, $P=0.328$; FAF, $P=0.231$; FA, $P=0.779)$. NIR detected $92.9 \%$ of the lacquer cracks, whereas FAF and FA imaging detected $12.5 \%$ and $67.9 \%$, respectively (we defined the ICGA detection rate as $100 \%$ for comparison). Although the detection rate of lacquer cracks using FA imaging was higher than that of FAF imaging, FA imaging had limitations in defining the location and size
Table 1 Demographic data for 56 eyes with lacquer cracks

\begin{tabular}{lccc}
\hline & $\begin{array}{c}\text { Linear LC } \\
\text { 26 eyes }\end{array}$ & $\begin{array}{c}\text { Stellate LC } \\
\text { 30 eyes }\end{array}$ & P-value \\
\hline Age (years) & $42.1 \pm 12.7$ & $46.4 \pm 9.2$ & 0.165 \\
Gender, male:female & $10: 16$ & $8: 22$ & 0.350 \\
Refractive error (diopters) & $-13.44 \pm 3.11$ & $-14.17 \pm 2.95$ & 0.328 \\
Axial length (mm) & $29.27 \pm 1.42$ & $29.65 \pm 1.37$ & 0.209 \\
& & & \\
Best-corrected visual acuity & & & \\
$\quad$ LogMAR & $0.55 \pm 0.62$ & $0.58 \pm 0.49$ & 0.409 \\
$\quad$ Snellen & $0.49 \pm 0.37$ & $0.40 \pm 0.29$ & 0.423 \\
\hline
\end{tabular}

Abbreviations: LC, lacquer crack; MAR, minimum angle of resolution. Data are shown as mean \pm standard deviation.

Table 2 Detection rate for lacquer cracks in different method

\begin{tabular}{lcccc}
\hline $\begin{array}{l}\text { LC detection } \\
(\%)\end{array}$ & $\begin{array}{c}\text { Linear LC } \\
26 \text { eyes }\end{array}$ & $\begin{array}{c}\text { Stellate LC } \\
30 \text { eyes }\end{array}$ & P-value & $\begin{array}{c}\text { Overall } 56 \text { eyes } \\
34 \text { patients }\end{array}$ \\
\hline NIR & $23(88.5 \%)$ & $29(96.7 \%)$ & 0.328 & $52(92.9 \%)$ \\
FAF & $5(19.2 \%)$ & $2(6.7 \%)$ & 0.231 & $7(12.5 \%)$ \\
FA & $17(65.4 \%)$ & $21(70.0 \%)$ & 0.779 & $38(67.9 \%)$ \\
ICGA & $26(100 \%)$ & $30(100 \%)$ & 1 & $56(100 \%)$ \\
\hline
\end{tabular}

Abbreviations: FA, fluorescence angiography; FAF, fundus autofluorescence; ICGA, indocyanine green angiography; LC, lacquer crack; NIR, near-infrared reflectance.

of the lacquer cracks compared with NIR imaging (example 2, Figure 1).

\section{Comparison of NIR and ICGA imaging in eyes with lacquer cracks}

Lacquer cracks appeared as hyperreflective lines on NIR, which corresponded to the hypofluorescent lines on late-phase ICGA (Figures 1 and 2). On NIR, we noticed some artifactual reflective spots that were quite different from the hyperreflective lines of the lacquer cracks (asterisks in Figure 2).

\section{Correlation of NIR and SD-OCT in eyes with lacquer cracks and patchy chorioretinal atrophy}

Horizontally and vertically oriented SD-OCT through the lacquer cracks showed a continuous retinal pigment epithelium-Bruch's membrane (RPE-BM) complex line, thinner choroids, and acoustic shadows. The thinner choroids and acoustic shadows correlated with the lacquer cracks, which appeared as hyperreflective lines on NIR imaging (asterisks in Figures $3 b-d$ ).

We also compared NIR and SD-OCT images of an eye with both lacquer cracks and patchy chorioretinal 

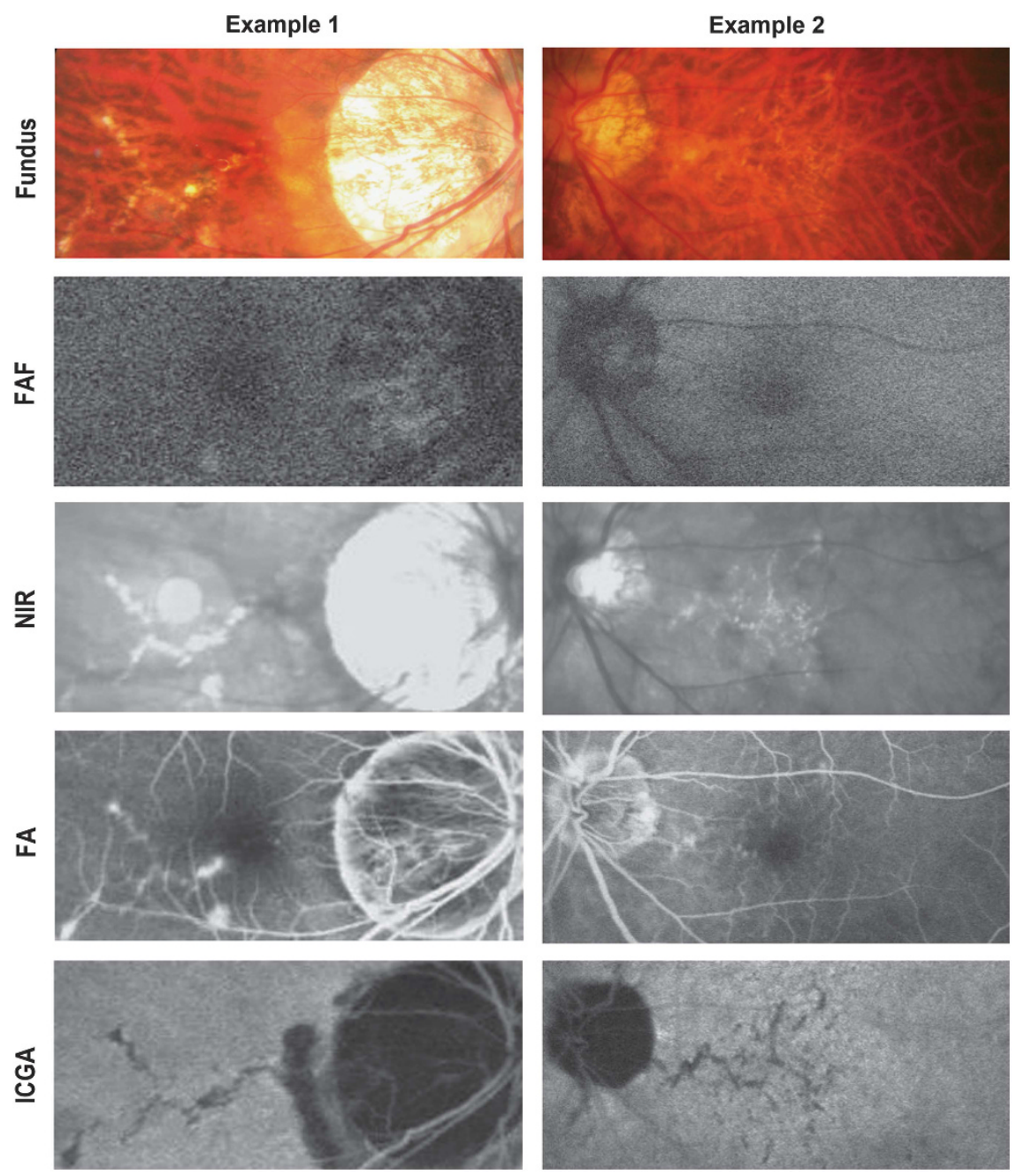

Figure 1 Multimodal imaging of the eyes with lacquer cracks. Fundus photographs of lacquer cracks showed yellowish lines and dots. FAF did not show the lesions of lacquer cracks. NIR revealed hyperreflective lines that correlated with the hypofluorescent lines on late-phase ICGA. FA showed a window defect that correlated with the lacquer crack lesion in example 1 (left), whereas FA did not show the extension and shape of the lacquer cracks in example 2 (right).

atrophy (Figure 3e). NIR showed hyperreflectance in the region of patchy atrophy (zone 3, Figure 3e) and the lacquer cracks (zone 2, Figure 3e), and ICGA showed hypofluorescence in both areas (Figure 3e). An SD-OCT scan through transitional changes from no lacquer cracks (zone 1, Figure 3e) to lacquer cracks (zone 2, Figure 3e), and then to patchy atrophy (zone 3, Figure 3e),

respectively revealed a continuous RPE-BM complex line (zone 1, Figure 3e), a continuous RPE-BM complex line with a thinner choroid (zone 2, Figure 3e), and the absence of an RPE-BM complex line and choroid (zone 3, Figure 3e).

\section{Discussion}

In our study, confocal NIR imaging appears to be an excellent noninvasive method for detecting lacquer cracks in vivo in highly myopic eyes, which can help guide clinicians in their use of ICGA. In addition, SD-OCT in combination with NIR located primary pathological lacquer cracks in the intact retinal pigment epithelium-Bruch's membrane complex as well as thinner choroid.

\section{Detection of lacquer cracks with multimodal retinal imaging}

Because FAF is mainly used to detect lipofuscin in the $\mathrm{RPE}^{21}$ the low rate of lacquer crack detection with FAF (12.5\%) may be related to a continuous RPE-BM line overlying the lacquer cracks. Cohort studies have shown that cataract is a complication of myopia, ${ }^{22}$ and that nuclear-type cataracts may absorb blue light $(488 \mathrm{~nm})$, which excites lipofuscin during FAF and the fluorescent 

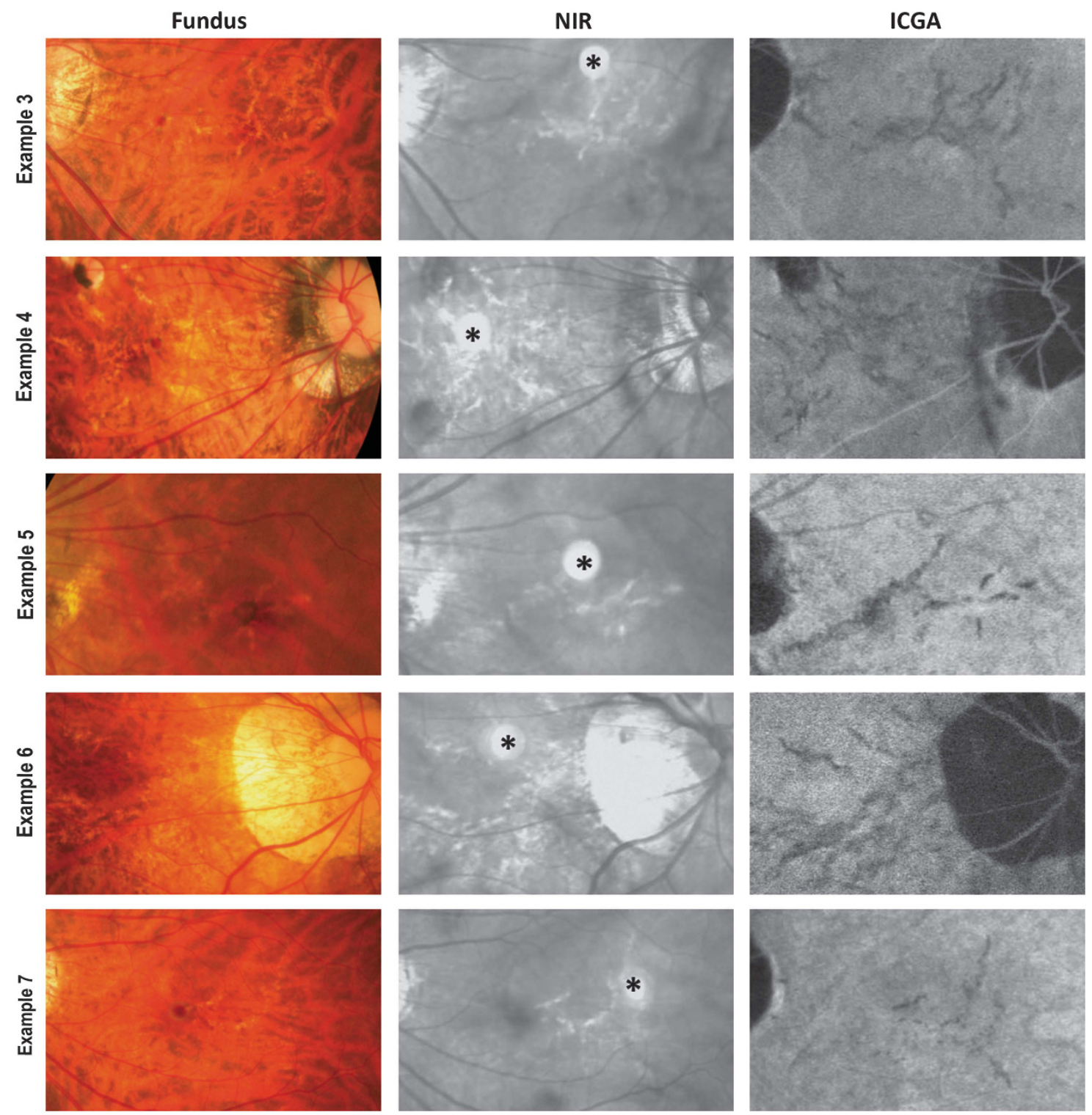

Figure 2 Correlation of fundus photography (left), NIR (middle), and ICGA (right) in the eyes with lacquer cracks. NIR showed hyperreflective lines corresponding to the hypofluorescent lines on ICGA. Artifactual reflective spots were evident on the NIR image (asterisks).

signal during FA. Taking advantage of the ART function built into the Heidelberg Retina Angiograph that enables the capture of multiple images in the exact same location, we can obtain better contrast and detail and eliminate noise caused by mild-to-moderate nuclear-type cataracts. However, in patients with severe cataracts, retinal images are more challenging to obtain, and as a result those patients were excluded from this study. As we have excluded severe cataract, the low rate of lacquer crack detection with FAF (12.5\%) in our study is unlikely to be related to cataract, but may be related to a continuous RPE-BM line overlying the lacquer cracks unless there are RPE changes nearby.
Near-infrared light not only penetrates the optical media more easily than FAF or FA, but also provides better visualization of the subretinal and choroidal structures than the light of the shorter wavelengths used for FAF and FA. ${ }^{16,23}$ Furthermore, NIR is less influenced by lens opacification than FAF or FA.

Nevertheless, the NIR images revealed some artifactual reflective spots (asterisks in Figure 2) that confounded the detection of lacquer cracks. However, because these spots were always present in the center of the image field, they could have been avoided by taking the NIR images from different angles, or by centering the optic nerve head so that the artifactual reflective 
a
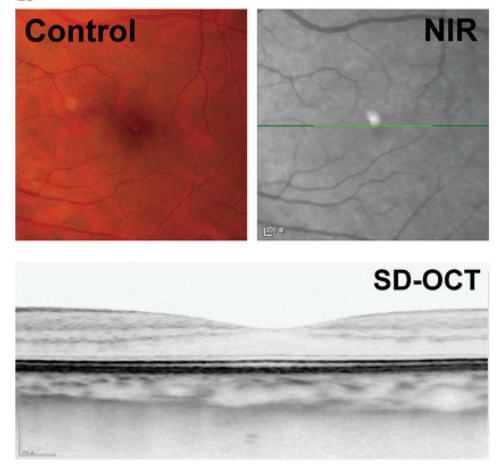

c
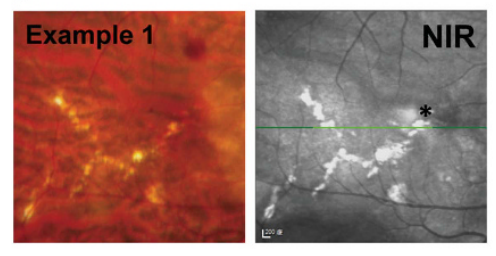

SD-OCT

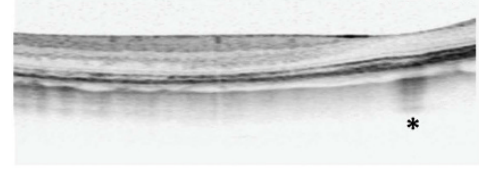

b
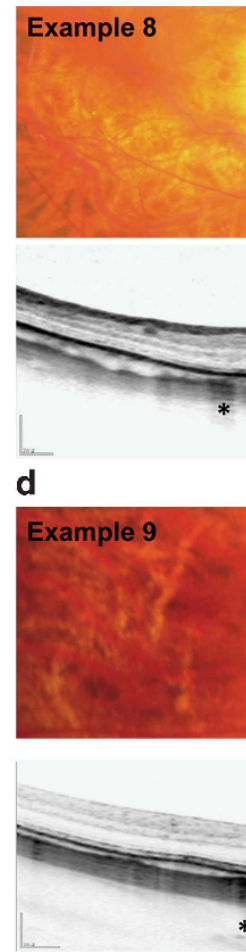

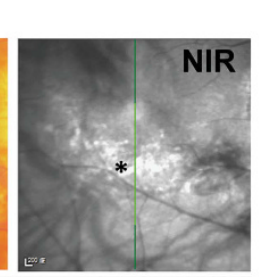

SD-OCT

e
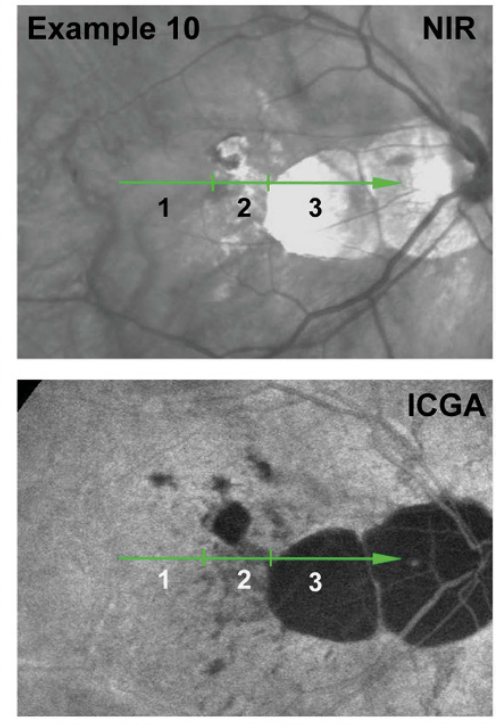

SD-OCT
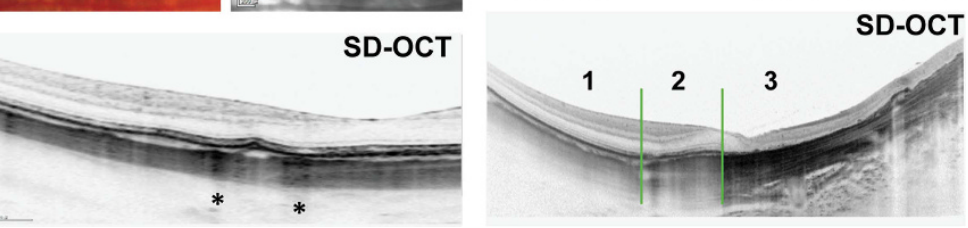

Figure 3 SD-OCT scans through the lacquer cracks in four patients with highly myopic eyes (b-e) and one emmetropic eye (control, a). (b-e), NIR showed hyperreflectant lines, and OCT scans revealed a continuous RPE-Bruch's membrane complex, with a thinner choroid and acoustic shadows (asterisks, (b-d)). (e) NIR of a highly myopic eye with patchy chorioretinal atrophy showed a hyperreflective patch (zone 3) and lines (lacquer cracks, zone 2). IGCA revealed hypofluorescence in the area of patchy atrophy (zone 3) and lacquer cracks (zone 2). SD-OCT analysis of the corresponding scanned section showed thinner choroid and a continuous RPE-BM complex (zone 2) compared with the area without a lacquer crack (zone 1), and an absence of RPE-Bruch's membrane complex and choroid in the area of patchy atrophy (zone 3).

spot does not affect the detection of a lacquer crack in the macular area.

\section{NIR of lacquer cracks and correlation with SD-OCT}

The hyperreflectance in the NIR images of lacquer cracks may be explained by one of two mechanisms or a combination of both. The first possible explanation attributes this bright reflectance of the lacquer cracks on NIR images to the reduced absorption of near-infrared light by oxygenated hemoglobin, which is caused by fibrotic choroidal vessels and/or an attenuated choriocapillaris. Elsner et $a l^{23}$ found that choroidal vessels are infrared light hyporeflective because of increased absorption by oxygenated hemoglobin. The reduced level of oxygenated hemoglobin in fibrotic choroidal vessels and/or attenuated choriocapillaris thereby reduces the absorption of infrared radiation, resulting in the hyperreflectance seen on NIR images. Fibrotic choroidal vessels and an attenuated choriocapillaris might also result in hypofluorescence on ICGA because the inner choroidal circulation is compromised and the permeation capacity of the ICG molecules is reduced.

The second possible explanation is that reflectance from the sclera is increased because of choroidal thinning. One eye in the current study had both lacquer cracks and patchy chorioretinal atrophy with this phenomenon (Figure 3e). The evidence of transitional changes as the SD-OCT scan moved from the lacquer cracks to the patchy atrophy indicates that patchy chorioretinal atrophy involves an area with greater thinning of the choroid than that which occurs in the area associated with lacquer cracks, and that NIR images show brighter reflectance in areas of patchy chorioretinal atrophy than in lacquer cracks. As indicated by other studies, $3,4,11$ patchy chorioretinal atrophy is probably a more advanced phase of myopic maculopathy than lacquer cracks. Similar NIR hyperreflectance correlated to a lack of choroid was observed in eyes from the patients with choroideremia (Figure 4a). 

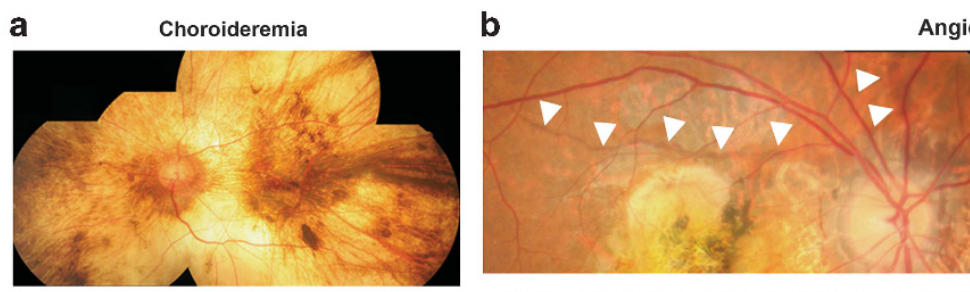

Angioid Streak
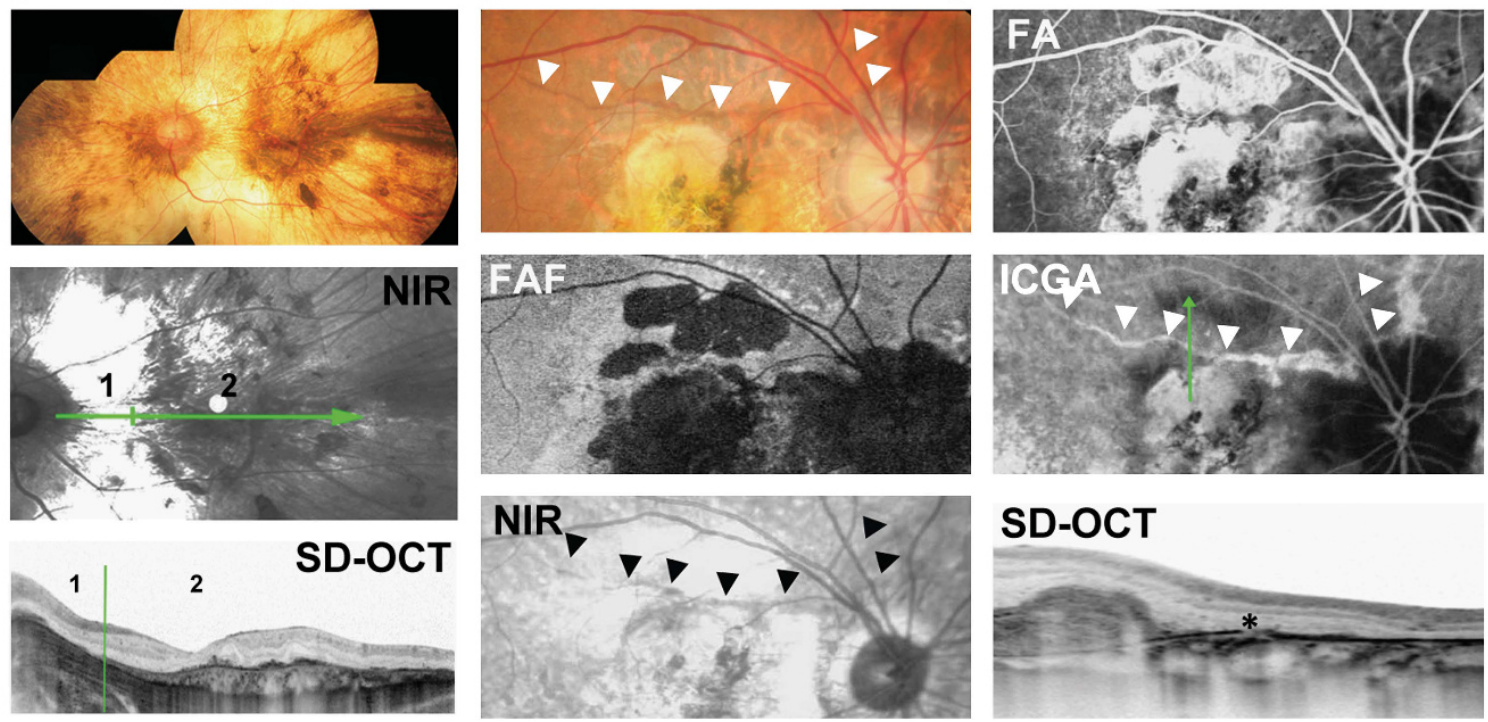

\section{SD-OCT}

Figure 4 Multimodal retinal imaging in the eyes with choroideremia and angioid streaks. (a) An example eye from a patient with choroideremia showed an area lacking choroid on SD-OCT (zone 1) correlated with the hyperreflective area on NIR (zone 1), whereas the area of relative intact choroid thickness on SD-OCT (zone 2) showed lower reflectance (zone 2) on NIR. (b) A fundus photograph of an angioid streak in an example eye from a patient with pseudoxanthoma elasticum showed dark lines. FAF and FA labeled the area with an RPE defect, but failed to localize the area of the angioid streak. Near-infrared reflectance revealed hyporeflective lines that correlated with the hyperfluorescent lines on late-phase ICGA. An SD-OCT image corresponding to the scanned section showed a break in RPE-Bruch's membrane complex (asterisks).

Ohno-Matsui et $a l^{6}$ performed an ICGA analysis of highly myopic eyes with retinal hemorrhage, and concluded that lacquer cracks represent a region of ruptured RPE-BM complex which has been completely repaired with scar tissue. In our study, SD-OCT showed a consistently continuous RPE-BM complex line in the lesions of the lacquer cracks, and the acoustic shadows (asterisks in Figures $3 b-d$ ) in the SD-OCT images of the lacquer cracks may have been related to fibrotic choroidal vessels. Consistent with the observations of Ohno-Matsui et al, ${ }^{6}$ we suggest that lacquer cracks represent a 'healed' lesion. Further studies on SD-OCT of highly myopic eyes with only macular hemorrhage but no $\mathrm{CNV}$ are required to determine whether there is a concomitant break in Bruch's membrane.

\section{Comparison of multimodal retinal imaging in eyes with lacquer cracks and angioid streaks}

Pruett et al. studied the pattern of break formation in lacquer cracks, angioid streaks, and traumatic tears in Bruch's membrane. ${ }^{24}$ They concluded that these three diseases have pathological similarities. ${ }^{24}$ Previous studies have shown that lacquer cracks represent breaks in the RPE-BM-choriocapillaris complex, ${ }^{9,11-13}$ and that their histopathological attributes are similar to those of angioid streaks. ${ }^{24}$ However, recent studies on angioid streaks in patients with pseudoxanthoma elasticum have reported that angioid streaks produce hyporeflectance on NIR and hyperfluorescence on late-phase ICGA, and that SD-OCT visualizes breaks in Bruch's membrane (Figure 4b). ${ }^{25}$ We hypothesize that the histopathological findings of lacquer cracks differ from those of angioid streaks on the basis of the different features revealed with NIR imaging, ICGA, and SD-OCT.

In conclusion, multimodal cSLO imaging in combination with SD-OCT is an excellent method to detect lacquer cracks in highly myopic eyes. In eyes with lacquer cracks without hemorrhage or CNV, the hyperreflectance of lacquer cracks seen on NIR images may be related to thinner choroids and/or fibrotic choroidal vessels, and/or an attenuated choriocapillaris. By using the multimodal cSLO imaging and SD-OCT analysis, we found that the RPE$\mathrm{BM}$ complex line is continuous in eyes with lacquer cracks. Nevertheless, the limitations of the current study are that it was an in vivo imaging study, and we only examined eyes with lacquer cracks in a stable condition, rather than lacquer cracks with retinal hemorrhage or subretinal CNV. Furthermore, SD-OCT studies and histopathological studies of highly myopic eyes without macular hemorrhage or $\mathrm{CNV}$ are required to provide further information about lacquer cracks. 


\section{Summary}

\section{What was known before}

- The best way to visualize lacquer cracks is with indocyanine green angiography (ICGA), which is an invasive examination. However, dye allergy is a concern when ICGA is used to detect lacquer cracks in highly myopic eyes.

- There are no histopathological studies on lacquer cracks without retinal hemorrhage or subretinal neovascularization.

\section{What this study adds}

- Multimodal confocal scanning laser ophthalmoscopy imaging in combination with spectral-domain optical coherence tomography is an excellent method to detect lacquer cracks in highly myopic eyes.

- Spectral-domain optical coherence tomography in combination with near-infrared reflectance located primary pathological lacquer cracks in the intact retinal pigment epithelium-Bruch's membrane complex as well as thinner choroid.

\section{Conflict of interest}

The authors declare no conflict of interest.

\section{Acknowledgements}

Publication of this article was supported in part by research grant NSC-102-2314-B-182A-102-MY3 from the Taiwan National Science Council, Taiwan, and CMRPG 391801-391803 and 3C1051 from Chang Gung Memorial Hospital, Linkou, Taiwan. The funders had no role in study design, data collection and analysis, decision to publish, or preparation of the manuscript. The authors thank the retinal imaging technicians (Department of Ophthalmology, Chang Gung Memorial Hospital, Linkou and Taipei) for their technical assistance. No acknowledgment of any proprietary interests or research funding when presenting some related work of this manuscript (as an invited speech) in the 8th APVRS Congress in Nagoya December 2013, and in online presentation, Macula Society 2014 in Key Largo, Florida.

\section{References}

1 Liu JH, Cheng CY, Chen SJ, Lee FL. Visual impairment in a Taiwanese population: prevalence, causes, and socioeconomic factors. Ophthalmic Epidemiol 2001; 8: 339-350.

2 Morgan IG, Ohno-Matsui K, Saw SM. Myopia. Lancet 2012; 379: $1739-1748$.

3 Hayashi K, Ohno-Matsui K, Shimada N, Moriyama M, Kojima A, Hayashi W et al. Long-term pattern of progression of myopic maculopathy: a natural history study. Ophthalmology 2010; 117: 1595-1611.
4 Ohno-Matsui K, Tokoro T. The progression of lacquer cracks in pathologic myopia. Retina 1996; 16: 29-37.

5 Ikuno Y, Sayanagi K, Soga K, Sawa M, Gomi F, Tsujikawa M et al. Lacquer crack formation and choroidal neovascularization in pathologic myopia. Retina 2008; 28: 1124-1131.

6 Ohno-Matsui K, Ito M, Tokoro T. Subretinal bleeding without choroidal neovascularization in pathologic myopia. A sign of new lacquer crack formation. Retina 1996; 16: 196-202.

7 Ohno-Matsui K, Yoshida T, Futagami S, Yasuzumi K, Shimada N, Kojima A et al. Patchy atrophy and lacquer cracks predispose to the development of choroidal neovascularisation in pathological myopia. $\mathrm{Br} J$ Ophthalmol 2003; 87: 570-573.

8 Wang NK, Lai CC, Chu HY, Chen YP, Chen KJ, Wu WC et al. Classification of early dry-type myopic maculopathy with macular choroidal thickness. Am J Ophthalmol 2012; 153: 669-677.

9 Salzmann M. The choroidal changes in high myopia. Arch Ophthalmol 1902; 31: 41-42.

10 Klein RM, Curtin BJ. Lacquer crack lesions in pathologic myopia. Am J Ophthalmol 1975; 79: 386-392.

11 Grossniklaus HE, Green WR. Pathologic findings in pathologic myopia. Retina 1992; 12: 127-133.

12 Avila MP, Weiter JJ, Jalkh AE, Trempe CL, Prutt RC, Schepens CL. Natural history of choroidal neovascularization in degenerative myopia. Ophthalmology 1984; 91: 1573-1581.

13 Klein RM, Green S. The development of lacquer cracks in pathologic myopia. Am J Ophthalmol 1988; 106: 282-285.

14 Ohno-Matsui K, Morishima N, Ito M, Tokoro T. Indocyanine green angiographic findings of lacquer cracks in pathologic myopia. Jpn J Ophthalmol 1998; 42 293-299.

15 Delori FC, Dorey CK, Staurenghi G, Arend O, Goger DG, Weiter JJ. In vivo fluorescence of the ocular fundus exhibits retinal pigment epithelium lipofuscin characteristics. Invest Ophthalmol Vis Sci 1995; 36: 718-729.

16 Keilhauer CN, Delori FC. Near-infrared autofluorescence imaging of the fundus: visualization of ocular melanin. Invest Ophthalmol Vis Sci 2006; 47: 3556-3564.

17 Wang NK, Lai CC, Chou CL, Chen YP, Chung LH, Chao AN et al. Choroidal thickness and biometric markers for the screening of lacquer cracks in patients with high myopia. PLoS One 2013; 8: e53660.

18 Wang NK, Chuang LH, Lai CC, Chou CL, Chu HY, Yeung L et al. Multimodal fundus imaging in fundus albipunctatus with RDH5 mutation: a newly identified compound heterozygous mutation and review of the literature. Doc Ophthalmol 2012; 125: 51-62.

19 Spaide RF, Koizumi H, Pozzoni MC. Enhanced depth imaging spectral-domain optical coherence tomography. Am J Ophthalmol 2008; 146: 496-500.

20 von Ruckmann A, Fitzke FW, Bird AC. Distribution of fundus autofluorescence with a scanning laser ophthalmoscope. Br J Ophthalmol 1995; 79: 407-412.

21 Schmitz-Valckenberg S, Holz FG, Bird AC, Spaide RF. Fundus autofluorescence imaging: review and perspectives. Retina 2008; 28: 385-409.

22 Lim R, Mitchell P, Cumming RG. Refractive associations with cataract: the Blue Mountains Eye Study. Invest Ophthalmol Vis Sci 1999; 40: 3021-3026. 
23 Elsner AE, Burns SA, Weiter JJ, Delori FC. Infrared imaging of sub-retinal structures in the human ocular fundus. Vision Res 1996; 36: 191-205.

24 Pruett RC, Weiter JJ, Goldstein RB. Myopic cracks, angioid streaks, and traumatic tears in Bruch's membrane. Am J Ophthalmol 1987; 103: 537-543.
25 Charbel Issa P, Finger RP, Holz FG, Scholl HP. Multimodal imaging including spectral domain OCT and confocal near infrared reflectance for characterization of outer retinal pathology in pseudoxanthoma elasticum. Invest Ophthalmol Vis Sci 2009; 50: 5913-5918. 